\title{
Transient electrical discharges in small devices*
}

\author{
Leopoldo Soto, ${ }^{\dagger}$ Andrey Esaulov, ${ }^{\text {a) }}$ José Moreno, Patricio Silva, Gustavo Sylvester, \\ Marcelo Zambra, Andrey Nazarenko, ${ }^{\text {b) }}$ and Alejandro Clausse ${ }^{c)}$ \\ Comisión Chilena de Energía Nuclear, Casilla 188-D, Santiago, Chile
}

(Received 27 October 2000; accepted 5 January 2001)

\begin{abstract}
Fundamental and applied research on plasmas with high energy density that are unstable and radiate can be done at a relatively low cost with small plasma pinches. In this paper we discuss three experiments using small pinch devices: a capillary discharge, a Z-pinch driven by a small generator, and a low energy plasma focus. The experiments were complemented by magnetohydrodynamics numerical calculations in order to assist the design and physical interpretation of the experimental data. The diagnostics used in the experiments include current and voltage monitors, multipinhole camera, holographic interferometry, and vacuum ultraviolet spectroscopy. (C) 2001 American Institute of Physics. [DOI: 10.1063/1.1351829]
\end{abstract}

\section{INTRODUCTION}

Transient electrical discharges are ideal ways to create interesting plasmas, particularly in small laboratories. Small plasma pinches can reproduce scenarios of high energy densities, radiation emission, and instability phenomena, which trigger fundamental and applied research with relatively low costs. A dynamic pinch is a transient plasma column conducting electrical current, which is confined by the associated magnetic field. Plasma pinches usually require the use of high pulsed voltages ( $\mathrm{kV}$ to $\mathrm{MV}$ ) and high currents (kA to MA). Generally, these devices operate by connecting a charged capacitor array to a pair of electrodes. Different electrode configurations have been proposed to create plasma pinches during pulsed discharges, Z-pinch, $\theta$ pinch, and plasma focus being the most well-known.

The applications of pinch discharges are mainly related to the radiation emitted by the plasma, namely electrons and ion beams, $x$ rays, and neutrons (using deuterium). Fast electrical discharges are also used to study plasma dynamics and stability. In this article three experiments are discussed, which use small devices to study fundamentals and applications of transient pinch electrical discharges: (a) a fast capillary discharge, (b) the early stages, dynamics and stability of a gas embedded Z-pinch, and (c) the development of a neutron pulsed source based on a small plasma focus.

\section{VUV RADIATION FROM CAPILLARY DISCHARGES}

In order to produce high brightness radiation with an electrical discharge, high energy densities should be provided to the plasma, $\sim 10^{12} \mathrm{~J} / \mathrm{m}^{3}$ (i.e., some $\mathrm{MJ}$ into a plasma

\footnotetext{
*Paper BI2 4, Bull. Am. Phys. Soc. 45, 20 (2000).

Invited speaker.

${ }^{a)}$ Present address: Institute of Theoretical and Experimental Physics, Moscow 117259, Russia and Laboratory for Plasma Astrophysics, Toyama University, 3190 Gofuku, Toyama 930-8555, Japan.

b) Also at the Institute of Spectroscopy, Russian Academic of Science, Troitsk, Moscow Region, 142090 Russia.

c) Also at the Network PLADEMA, Comisión Nacional de Energía Atómica and Universidad Nacional del Centro, 7000 Tandil, Argentina.
}

column of a centimeter in length and radius). This is usually done by means of huge facilities such as the Sandia National Laboratory in the USA. ${ }^{1,2}$ Intermediate devices have been used to produce vacuum ultraviolet (VUV) to soft $\mathrm{x}$-ray radiation $^{3,4}$ the most well-known being soft $\mathrm{x}$-ray lasing. ${ }^{4,5}$ Interestingly, $10^{12} \mathrm{~J} / \mathrm{m}^{3}$ can also be provided using small devices. In effect, by confining the plasma to submilimetric volumes, less than $1 \mathrm{~J}$ is required to reach high energy densities (as an extreme example $0.1 \mathrm{~J}$ is enough for a $60 \mu \mathrm{m}$ diameter sphere). The key point to produce those conditions in small devices is the current rate, which should reach about $10^{13} \mathrm{~A} / \mathrm{s} .{ }^{6}$ Consequently, the inductance should be very low.

We studied the physical mechanisms related to laser emission in the VUV to soft x-ray region in a table-top device. A fast capillary discharge was constructed at the Comisión Chilena de Energía Nuclear, CCHEN, following the design reported by Choi and Favre $(0.1-1 \mathrm{~J}, d I / d t$ $\left.\geqslant 10^{12} \mathrm{~A} / \mathrm{s}\right){ }^{7}$ The apparatus is shown in Fig. 1. A pair of 90 $\mathrm{mm}$ diameter brass electrodes from the anode and the cathode of the discharge, as well as a parallel plate capacitor. The insulator (Mylar and polyvinylidenefluoride, PVDF) between the electrodes is also the capacitor dielectric. A $1 \mathrm{~mm}$ inner diameter capillary tube, $0.8-2.5 \mathrm{~mm}$ long, is placed at the axis. The discharge is produced inside the capillary. A primary $7 \mathrm{nF}$ capacitor charges the storage capacitor by means of a pulse. The length of the cable connections between the spark gap and the capacitor of the main discharge is calibrated to obtain the required timing between the discharge and the diagnostics. The ionization in the interelectrode space is assisted by a high-energy electron beam, which is originated in plasma inside the hollow cathode region. ${ }^{7-9}$ The discharge operates in argon with a cathode pressure of 100-600 mbar. The anode pressure is around five times less than the cathode pressure. Current rates about $10^{12} \mathrm{~A} / \mathrm{s}$ were obtained applying voltages of $10 \mathrm{kV}$.

The voltage in the capillary capacitor, the current, and the current derivative were measured in our experiments. A coaxial cable was biased at $-200 \mathrm{~V}$ and located in the discharge axis, behind the anode, in order to detect the impact of electrons (negative signal) or photons with energy greater 


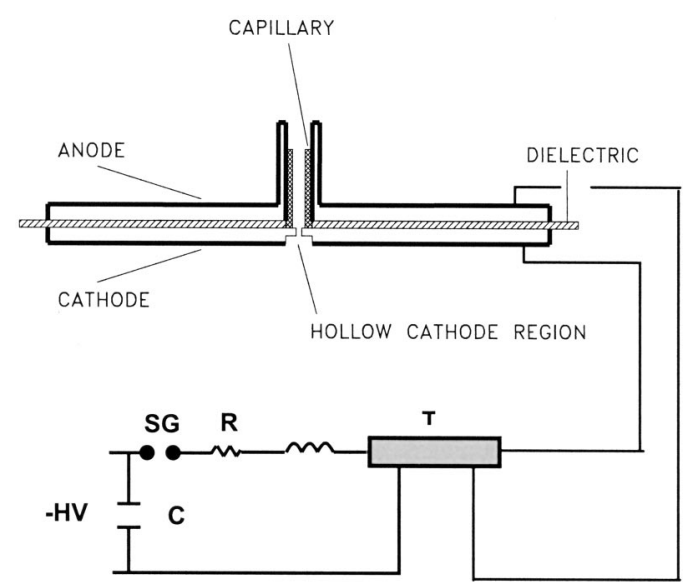

FIG. 1. Capillary discharge device. A pair of $90 \mathrm{~mm}$ diameter brass electrodes form the anode and the cathode of the discharge, as well as a parallel plate capacitor. A capillary of sub-millimeter radius and with a length of some centimeters, is placed at the axis. The discharge is produced inside the capillary between the capacitor plates. To operate the discharge, a direct current charged primary capacitor of $C=7 \mathrm{nF}$ is used to charge the storage capacitor with a pulse. From the spark gap SG, the capacitor of the main discharge is connected to three coaxial cables in parallel $(T)$.

than $4.3 \mathrm{eV}$ (positive signal, like a x-ray diode). A multipinhole camera with a magnification of $m=3$, attached to a microchannel plate (MCP) sensitive to $\mathrm{x}$ rays and VUV radiation was used to register 1 frame every 4 ns (Fig. 2). The VUV spectra of the capillary discharge have been obtained by means of a grazing incidence spectrograph GISVUV1-3S (Fig. 3). A gold-coated replica grating of 300 lines $/ \mathrm{mm}$, with $1 \mathrm{~m}$ curvature radius, was used. The grazing angle was $4^{\circ}$. The spectrograph was calibrated with helium for the spectral range of interest (20 to $200 \mathrm{~nm}$ ).

Figure 4 shows typical signals of voltage, current, and diode detector of a discharge along $8 \mathrm{~mm}$ length capillary and 650 mbar argon cathode pressure, at approximately -10 $\mathrm{kV}$ charging voltage. Time $t=0$ indicates the breakdown that occurs at $-10 \mathrm{kV}$. The peak current achieved was $5 \mathrm{kA}$ in $3.5 \mathrm{~ns}$, i.e., a half period of $7 \mathrm{~ns}$ in agreement with the approximately $2 \mathrm{nH}$ inductance of the $8 \mathrm{~mm}$ long capillary. With a capillary three times longer $(25 \mathrm{~mm})$ the half period of the discharge increases by $70 \%$, to $6 \mathrm{~ns}$, in accordance to an inductance three times longer, demonstrating that the pulsed power has an appropriately low inductance on the order of $\mathrm{nH}$. Negative signals were observed in the diode

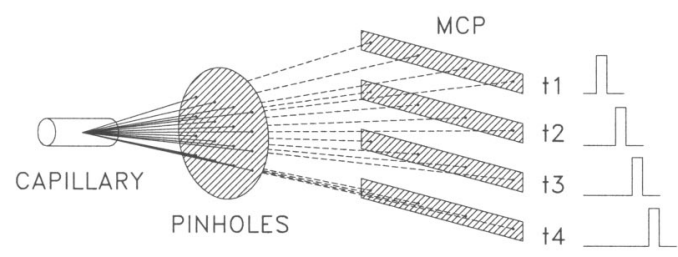

FIG. 2. Multipinhole camera. A four frame microchannel plate (MCP) was used to register images: one frame every $4 \mathrm{~ns}$. The MCP is sensitive only to $\mathrm{X}$-ray and VUV radiation. The gating pulse is $5 \mathrm{~ns}$. A square array of 16 pinholes was used; 4 columns with equal size pinholes per column: 50, 100, 200 , and $300 \mu \mathrm{m}$, respectively. The four rows or the pinhole array correspond to different frames separated by 4 ns. So, time-space resolution plasma images can be obtained.

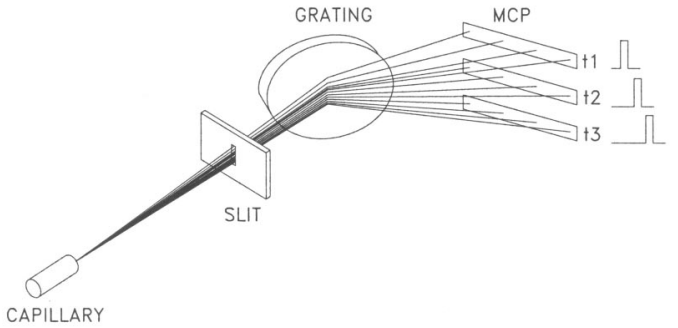

FIG. 3. VUV spectrograph. VUV spectra of the capillary discharge has been obtained by means of a grazing incidence spectrograph GISVUV1-3S. Gold-coated replica grating of 300 lines $/ \mathrm{mm}$, with a curvature radius of $1 \mathrm{~m}$ was used. The grazing angle was $4^{\circ}$. The spectra was registered in the same four MCP frames used with the pinhole camera (4 ns between frames and 5 ns gating pulse). Due to the geometry defined by the spectrograph, only three frames are used.

detector before breakdown (Fig. 4), indicating electron beams. About $3 \mathrm{~ns}$ after the peak current there were indications of photon radiation impacting on the diode, which produced positive signals. Pinhole images taken during a discharge along an $8 \mathrm{~mm}$ length capillary and $650 \mathrm{mbar}$ are shown in Fig. 5. It is important to note that the pinholes are not in the capillary axis, so some considerations must be taken into account. From simple geometry it is clear that images created from opposite ends of the capillary have different magnifications, and are centered in a different posi-

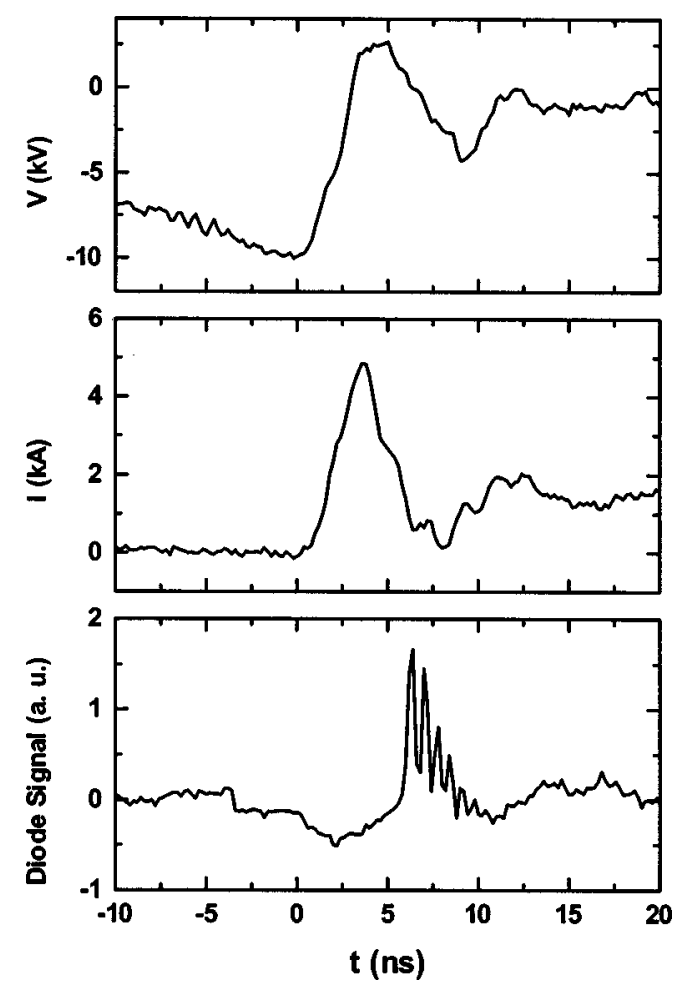

FIG. 4. Signals of voltage, $V$, current, $I$, and from a $-200 \mathrm{~V}$ biased diode detector corresponding to a capillary discharge in argon at 500 mTorr cathode pressure, $8 \mathrm{~mm}$ capillary length, and $1 \mathrm{~mm}$ diameter. Time $t=0$ correspond to the breakdown that was at $-10 \mathrm{kV}$. The maximum current achieved was $5 \mathrm{kA}$ with a rise time $10-90 \%$ less than $3 \mathrm{~ns}$. From the diode detector a negative signal appears before the breakdown, corresponding to the electron beam. Later, a positive signal appears corresponding to the photonic radiation impacting on the diode, 3 ns after the peak current. 


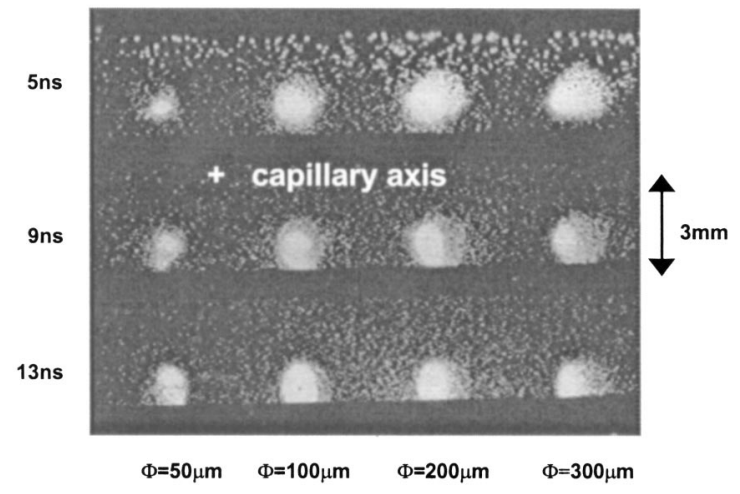

FIG. 5. Pinhole images corresponding to a capillary discharge at $500 \mathrm{mTorr}$ cathode pressure, a capillary of $8 \mathrm{~mm}$ in length, and $1 \mathrm{~mm}$ in diameter. The cross indicates the position of the capillary axis. Unfortunately, only 3 of the 4 frames of MCP were working at that time.

tion. Thus, for a qualitative analysis three possibilities were considered. For a radial homogeneous plasma inside the capillary, the image corresponds to nonconcentric circles [Fig. 6(a)]. In a hollow plasma column, the image has a more complex structure [Fig. 6(b)]. In a compressed plasma, the image becomes a line [Fig. 6(c) $].{ }^{10}$ According to that interpretation, in Fig. 5, the images for 5 ns $(2 \mathrm{~ns}$ after the peak current) suggest the existence of a homogeneous plasma column. For the $\Phi=50 \mu \mathrm{m}$ pinhole diameter, a central brightness core of $\sim 300 \mu \mathrm{m}$ can be observed, which is not necessarily a compressed plasma.

Figure 7 shows a time-integrated spectrum over ten discharges along an $8 \mathrm{~mm}$ length capillary (a single shot did not have enough intensity under these conditions to register a spectrum). Several lines are attributed to ArVIII, ArIX, and

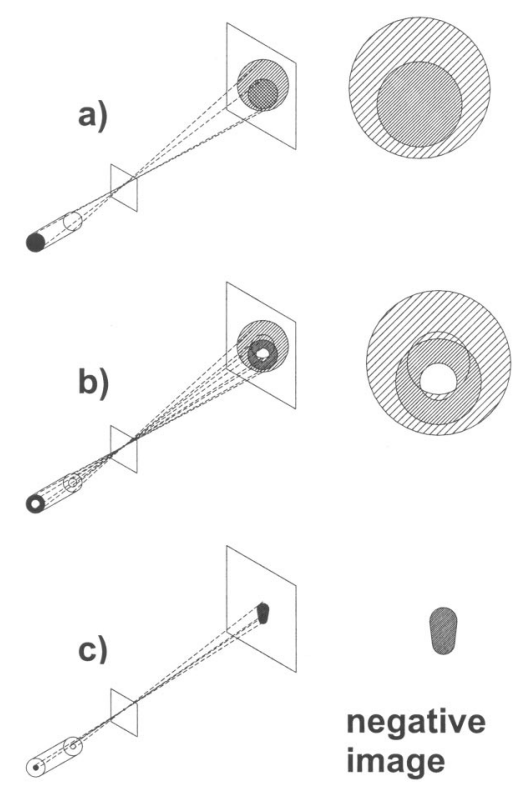

FIG. 6. Qualitative images obtained with a pinhole not in the capillary axis. Images created from opposite ends of the capillary have different magnifications, and are centered on a different position. (a) In the case of a radial homogeneous plasma, the image corresponds to nonconcentric circles. (b) In a hollow plasma column, the image has a more complex structure. (c) In a compressed plasma, the image becomes a line (Ref. 12).

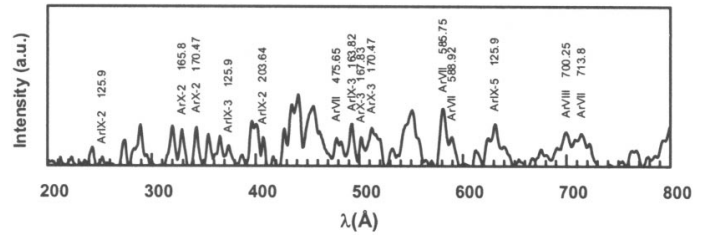

FIG. 7. Time integrated spectrum: 10 shots were registered in the same frame of the MCP for a discharge with a capillary $8 \mathrm{~mm}$ in length and $1 \mathrm{~mm}$ in diameter.

ArX ions (second and higher orders can be identified: ArIX-2, ArIX-3, ArIX-5). The ion temperature can be roughly estimated at about $40-60 \mathrm{eV}$.

The capillary discharge can also be complemented with numerical simulations. A one-dimensional model derived from the magnetohydrodynamics (MHD) equations was applied to study the plasma dynamics and temperature evolution during the discharges. ${ }^{11}$ The calculations predict a final radius of 100-200 microns and electron densities of 2 $\times 10^{24} \mathrm{~m}^{-3}$ occurring three nanoseconds after the peak current. The corresponding electron and ion temperatures are 80 and $40 \mathrm{eV}$. Under these conditions, theoretically soft $\mathrm{x}$-ray lasing can occur at $46.9 \mathrm{~nm}$, due to population inversion by collisional electron excitation of Ne-like ArIX ions.

\section{GAS EMBEDDED Z-PINCH DRIVEN BY A SMALL GENERATOR: EARLY STAGE, PLASMA DYNAMICS AND STABILITY}

Z-pinch discharges are very simple devices that can produce hot dense plasmas. The limitation is the discharge disruption by instabilities. The development of pulse power generators, capable of delivering currents to a plasma load of $I>10^{5} \mathrm{~A}$ with $d I / d t>10^{12} \mathrm{~A} / \mathrm{s}$ led to a widely held belief that by heating and compressing the plasma at a high enough rate, dense hot plasma conditions could be achieved in times shorter than the instability growth time. ${ }^{12}$ A number of experiments have been carried out in Z-pinches using pulse power generators. Results obtained show that in fiber and compressional Z-pinches $m=0$ magnetohydrodynamic (MHD) instabilities are present. On conventional gas embedded Z-pinches, after an initial expansion, $m=1$ instabilities leading to a helix develop, eventually disrupting the plasma column. The development of these instabilities happen during tens of nanoseconds. The observed growth time for the instabilities appearing in Z-pinch discharges, in both gas embedded Z-pinch ${ }^{13}$ and fiber ${ }^{14}$ or compressional is longer than those predicted by MHD theory. In a gas embedded Z-pinch where an electric discharge between the electrodes is established in a dense gas under atmospheric range pressure it is possible to obtain a large number of experiments per day in comparison with single fiber or fiber array experiments. In addition, changing the filling pressure and electrode configuration, different initial conditions and different stability regimes can be studied.

In this section we describe a series of experiments in gas embedded Z-pinches carried out at Pontificia Universidad Católica de Chile, ${ }^{15-18}$ driven by a small pulse power generator, a Marx bank $(400 \mathrm{kV})$ coupled to a water transmis- 

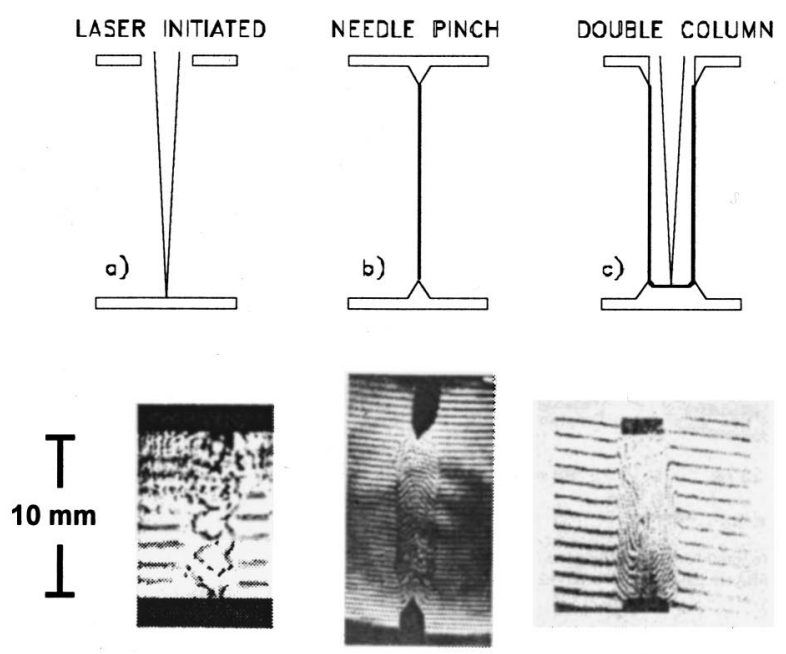

$45 \mathrm{~ns}$

56 ns
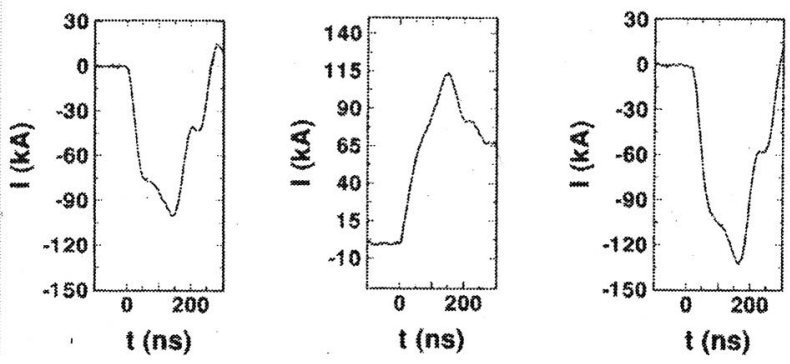

FIG. 8. $\mathrm{H}_{2}$ at $1 / 3$ bar gas embedded Z-pinch experiments. (a) Pre-ionization by the laser pulse (laser initiated pinch), (b) pre-ionization by microdischarge (needle pinch), (c) pre-ionization by the hollow microdischarge and laser pulse (double column pinch). Current traces and interferograms are shown.

sion line $(1.5 \Omega, 300 \mathrm{kV}, 120 \mathrm{~ns}$ double transit time). The current rate was approximately $2 \times 10^{12} \mathrm{~A} / \mathrm{s}$. The discharges were performed in $\mathrm{H}_{2}, \mathrm{D}_{2}$, and $\mathrm{He}$ at $1 / 6,1 / 3$, and 1 bar.

The electron density profile $n_{e}$, the line electron density $N$ (number of electron per unit length), and the pinch radius $a$, were measured with good temporal and spatial resolution. A frequency doubled Nd-YAG laser pulse (6 ns) was used for optical diagnostics to obtain multiframe holographic interferometry ( 8 frames separated by $10 \mathrm{~ns}$ between them), ${ }^{19}$ and to obtain simultaneous single shot image-plane holographic interferometry and shadowgraphy. A visible streak camera provided radial and axial plasma motion. ${ }^{18}$ The total current and the external voltage were also measured with a Rogowskii coil and a capacitive divider.

Figure 8 shows interferograms and current traces obtained in $\mathrm{H}_{2}$ at $1 / 3$ bar. The gas was pre-ionized using three different methods. The first method was based on laser induced ionization [Fig. 8(a), laser initiated pinch]. A pulse laser beam (Nd-YAG, $6 \mathrm{~ns}, 300 \mathrm{~mJ}$ ) was focused axially through the hollow anode onto the cathode a few nanoseconds before delivering the generator pulse to the electrodes. The size of the laser spark was less than $1 / 10$ of the interelectrode distance. The experiments showed that the plasma column expands in a radial direction during the first $25 \mathrm{~ns}$ $\left(\nu_{r}=1.7 \times 10^{4} \mathrm{~m} / \mathrm{s}\right)$, and then developed $m=1$ instabilities.
The electron density at the axis $n_{e} \sim 10^{24} \mathrm{~m}^{-3}$ was less than the filling gas density $\left(\sim 1.8 \times 10^{25} \mathrm{~m}^{-3}\right) .{ }^{17,18}$ The Bennett temperature $T_{\mathrm{B}}$, can be estimated from $T_{\mathrm{B}}=\left[\mu_{0} / 16 \pi k(Z\right.$ $+1)] I^{2} / N$, with $\mu_{0}$ the permeability of free space, $k$ the Boltzmann constant, and $Z$ the number of charges or ionization stages. Thus $T_{\mathrm{B}}(\mathrm{eV})=1.56 \times 10^{11} I^{2} / N$, with $I$ and $N$ in SI units. At $25 \mathrm{~ns}$ the Bennett temperature is estimated at $T_{\mathrm{B}} \sim 90 \mathrm{eV}$. While the plasma column expanded, the line density, $N$, increased roughly in a linear way, with a rate on the order of $4 \times 10^{25} \mathrm{~m}^{-1} \mathrm{~s}^{-1}( \pm 30 \%)$.

In the second method, a small $20 \mu \mathrm{A}$ discharge was induced between two tungsten needle electrodes [Fig. 8(b), needle pinch]. Here also the plasma column expanded $\left(\nu_{r}\right.$ $=2.6 \times 10^{4} \mathrm{~m} / \mathrm{s}$ ) during the current rise stage. No instabilities were observed during the expansion. ${ }^{16,18}$ A plume occupying $1 / 3$ of the electrodes interspace was observed at $5 \mathrm{~ns}$. An $n_{e}$ value of $5 \times 10^{25} \mathrm{~m}^{-3}$ is observed at $50 \mathrm{~ns}$ at the axis of the pinch, with a value of $N$ of $\sim 1.5 \times 10^{19} \mathrm{~m}^{-1}$, and the Bennett temperature was estimated at $T_{\mathrm{B}} \sim 40 \mathrm{eV}$. Line density, $N$, increased with a rate of $2.8 \times 10^{26} \mathrm{~m}^{-1} \mathrm{~s}^{-1}( \pm 30 \%)$. In this case, electron densities greater than what was expected according to the density of filling gas were measured.

In the third scheme, a cylindrical hollow discharge of $150 \mu \mathrm{A}$ was established between the conical electrodes, as well, a pulse laser beam was used for pre-ionization of the central zone ${ }^{17}$ [Fig. 8(c), double column pinch]. When the pulse voltage was applied, an initial $10 \mathrm{~ns}$ fast radial expansion phase was followed by about a $50 \mathrm{~ns}$ expansion of the central channel with a velocity of $\sim 2.6 \times 10^{4} \mathrm{~m} / \mathrm{s}$. The expansion of the internal boundary of the annular plasma was of the same order, whereas its external boundary had a slower expanding velocity $\sim 6 \times 10^{3} \mathrm{~m} / \mathrm{s}$. At the end of these expansion phases, two initial plasmas coalesced into the single plasma column with an apparent internal structure that was maintained during the next 110 ns. The line density, $N$, increased with a rate of the order of $1 \times 10^{26} \mathrm{~m}^{-1} \mathrm{~s}^{-1}$ $( \pm 30 \%)$ in the earliest $80 \mathrm{~ns}$, thus the rate decreased. The $n_{e}$ profiles after the $70 \mathrm{~ns}$, and until the current peak time, showed a central peak, with a maximum $n_{e}>4 \times 10^{25} \mathrm{~m}^{-3}$ and a value of $N$ of $\sim 5 \times 10^{19} \mathrm{~m}^{-1}$, and the Bennett temperature was estimated at $T_{\mathrm{B}} \sim 40 \mathrm{eV}$. The value of $n_{e}$ was greater than would be expected from the filling gas density. The size of the laser spark was less than $1 / 10$ of the interelectrode distance at $20 \mathrm{~ns}$, and no plasma plume was observed from the hollow electrodes. Double column pinch configuration discharges were characterized by low expansion rates and high electron densities, being, in general, more stable. $^{17,18}$

Bennett equilibrium (quasistatic or stationary pinch) has been assumed because the Alfvén velocity, $\nu_{\mathrm{A}}$, was greater than radial velocity in these three configurations.

The curious feature observed in the experiments described above was that the pinch radii always expanded ("no pinch effect'). This effect was rather surprising, and contradicts previous theoretical model calculations (see, for example, a zero-dimensional model in Refs. 20, 21). Actually, the models mentioned were derived assuming constant electron line density, while in these experiments the line density 


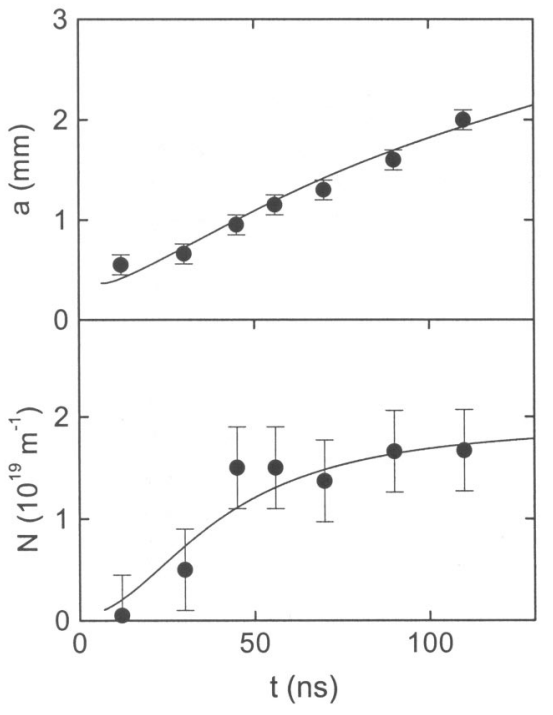

FIG. 9. Evolution of the pinch radius, $a$, and the electron line density, $N$, of a $\mathrm{H}_{2}$ at $1 / 3$ bar gas embedded Z-pinch (needle pinch). The experimental points were obtained from interferograms (Refs. 15 and 16). The curves are theoretical calculations. Radial evolution was obtained with a zerodimensional model including the contributions of the plasma density in the energy balance, continuity equation, and Bennett relation.

was always increasing. It is possible to develop an extended zero-dimensional model including the contributions of the time dependent plasma line density in the energy balance, continuity equation, and Bennett relation, ${ }^{15}$ which shows an excellent agreement with the experimental data (Fig. 9).

The relevant parameters regarding stability properties can be estimated from measured line density $N$, current $I$, and radius $a .^{22}$ In fact, assuming Bennett equilibrium, Haines and Coppins ${ }^{22}$ found for the Larmor radius over pinch radius $a_{i} / a$, for the transient Alfvén time $\iota_{A}=a / v_{\mathrm{A}}$, where $v_{\mathrm{A}}$ is the mean Alfvén speed, and for Lundquist number, $S$ :

$$
\begin{aligned}
& \frac{a_{i}}{a}=\left(\frac{4 \pi m_{i}}{\mu_{0} e^{2}}\right)^{1 / 2} \frac{1}{Z(1+Z)^{1 / 2}} \frac{1}{N^{1 / 2}}, \\
& \iota_{A}=\frac{a}{v_{\mathrm{A}}}=4\left(\frac{\pi m_{i}}{\mu_{0}}\right)^{1 / 2} a \frac{N^{1 / 2}}{I}, \\
& S=\frac{3 \mu_{0} \epsilon_{0}^{2} 2^{3 / 4}}{64\left(2 \pi m_{e} m_{i}\right)^{1 / 2} e^{2} \ln \Lambda Z(1+Z)^{3 / 2}} \frac{I^{4} a}{N^{2}},
\end{aligned}
$$

where $m_{e}$ and $m_{i}$ are the electron and ion mass, $Z$ is the number of charge or ionization stage, $\epsilon_{0}$ and $\mu_{0}$ are the permitivity and permeability of free space. Thus for hydrogen is obtained $a_{i} / a=5.7 \times 10^{8} \mathrm{~N}^{1 / 2} ; \quad \iota_{A}=2.6$ $\times 10^{-10} a N^{1 / 2} I^{-1} ; S=5.46 \times 10^{23} I^{4} a N^{-2}$. The number $\ln \Lambda$ $=5.36$ has been used. ${ }^{15}$

In order to determine if the plasma is in the magnetized regime the relevant parameter to examine is the product of ion cyclotron frequency $\Omega_{i}$ by collision time for the ions $\iota_{i}$,

$$
\Omega_{i} \tau_{i}=\frac{3 \mu_{0}^{5 / 2} \epsilon_{0}^{2} 2^{3 / 2}}{64\left(2_{e} m_{i}\right)^{1 / 2} e^{3} \ln \Lambda Z^{3}(1+Z)^{3 / 2}} \frac{I^{4} a}{N^{5 / 2}} .
$$

For $H_{2}, \Omega_{i} \iota_{i}=5.15 \times 10^{30} I^{4} a N^{-5 / 2}$.
Using the experimental measurements it is possible to evaluate these parameters. For the three pre-ionization schemes considered, from the value $\Omega_{i} \iota_{i}, 0.1-1$, it is apparent that the pinches are at the boundary of unmagnetized and magnetized regimes. The needle pinch and double column pinch remain stable during many transient Alfvén times $(\sim 20 \mathrm{~ns}$ at $\sim 80 \mathrm{~ns})$. It has been theoretically conjectured that there is a threshold for the stabilization due to resistive effects, corresponding to $S \sim 100$. Experimentally, it has been observed that for Z-pinch discharges with a substantially lower value of $S$, no instabilities appear. From the values of $S$, obtained at an early stage, it is apparent that the three discharges are resistive at early stages. In the laser initiated pinch case, when the instability $m=1$ starts, the value of $S$ is on the order of 150; later the value increases. The value observed for $a_{i} / a$ is at a later stage on the order of 0.1 -0.2 for the needle pinch and double column pinch. This is consistent with theoretical studies which indicate that the region of minimum instability for pinch discharges is in the neighborhood of $a_{i} / a=0.2$. $^{23,24}$

All the various configurations studied are initially in the resistive regime. Based on experimental observations in the double column pinch and in the needle pinch, ${ }^{25}$ it would appear that a pinch could be maintained stable if it crosses over the $S \sim 100$ boundary with a value $a_{i} / a$ around 0.1 -0.2 and remained at this value while the current is increasing.

To study the internal structure of the pinch, a onedimensional two-temperature MHD model was numerically analyzed. ${ }^{26}$ Interestingly, the results showed that the structure of the initially pre-ionized region greatly affects pinch dynamics coinciding with the experiments described above. There is still lot of are for further research in small Z-pinches, and it is clear that the advances will come from an intelligent partnership between theoretical and experimental work.

\section{SMALL PLASMA FOCUS IN THE LIMIT OF VERY LOW ENERGY}

Plasma focus is a pinch discharge produced between two coaxial electrodes. The discharge starts over the insulator surface [Fig. 10(a)]; the current sheath is magnetically accelerated along the coaxial electrodes [Fig. 10(b)], and after the current sheath runs over the ends of the electrodes a radial compression of the plasma occurs [Fig. 10(c)]. The final pinch generates beams of ions and electrons, and ultra short $\mathrm{x}$-ray pulses. Using deuterium gas, plasma focus devices produce fusion D-D reactions, generating a pulse of fast neutrons and protons.

During the last thirty years substantial efforts and resources have invested in plasma focus devices. ${ }^{27-30}$ The studies range from small devices around hundreds of Joules to large facilities about $1 \mathrm{MJ}$. Recently, plasma focus called the attention of the plasma research community, to develop pulsed radiation applications.

Here we describe a small plasma focus operating in the limit of very low energy $(\sim 100 \mathrm{~J})$. The plasma dynamics in this limit (low capacitance) are faster (low inductance) than 


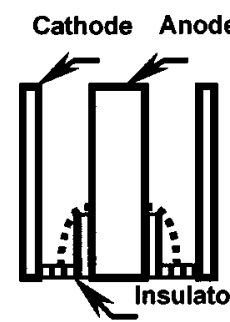

(a)

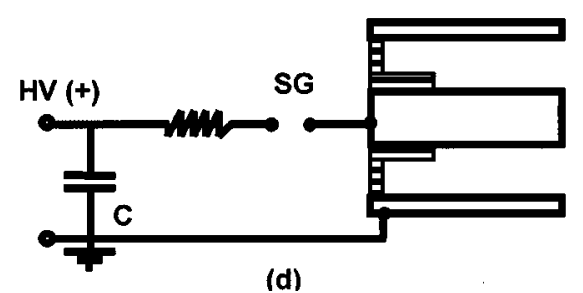

(d)

FIG. 10. Schematic drawing of the principal stages in the plasma dynamics in a plasma focus device. (a) The discharge starts in the base of the electrodes, a current sheath is formed at the insulator surface; (b) the current sheath is accelerated along the coaxial electrodes, (c) and a radial compression of the plasma occurs after the current sheaths reaches the axial end of the electrodes. (d) Scheme of the circuit, a capacitor $C$, is discharged over the electrode through a spark gap SG.

in large devices. Although in the small plasma focus the neutron yield per pulse is quite low $\left(10^{4}-10^{5}\right)$, it is possible to operate the device in a repetitive regime from $\mathrm{Hz}$ to $\mathrm{kHz}$.

The procedure to design a small plasma focus is to start with the available electrical components. In our case we had four capacitors $(40 \mathrm{nF}, 20 \mathrm{nH})$ connected in parallel $(160 \mathrm{nF}$, $5 \mathrm{nH})$. Charging voltages of 30 to $40 \mathrm{kV}$ could be obtained from a power supply or an intermediate pulse voltage multiplier. ${ }^{31}$ The optimum size of the electrodes was then determined by theoretical considerations, using a theoretical model of Mather plasma focus. ${ }^{32}$ Figure 11 shows the expected neutron yield dependence with the deuterium filling pressure for electrodes of length $z=2 \mathrm{~cm}$, internal and external radii of $r_{1}=0.8 \mathrm{~cm}$ and $r_{2}=1 \mathrm{~cm}$. An external inductance of $50 \mathrm{nH}$ was assumed. It can be seen that there is an optimum pressure that depends on the charging voltage.

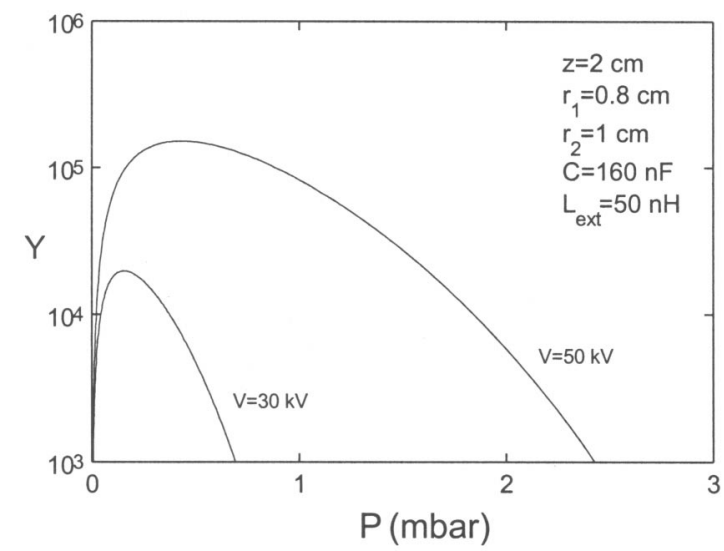

FIG. 11. Expected neutron yield, $Y$, for a electrode configuration of a length of $z=2 \mathrm{~cm}$, internal radius $r_{1}=0.8 \mathrm{~cm}$, and external radius $r_{2}=1 \mathrm{~cm}$. The discharge is assumed to be operating in deuterium with a total external inductance of $50 \mathrm{nH}$.
An important practical issue is the inductance of the circuit, which should be kept as low as possible. In small devices the inductance strongly depends on the length of the connections. In our case, we minimized the length of the connections between capacitor bank, spark gap, and electrodes using a compact configuration. A total external inductance of $60 \mathrm{nH}$ was measured.

The device constructed by us is useful both for basic research and applications. Possible diagnostics are time integrated and time resolution neutron and x-ray detection, PIN diode and particles detectors, pulsed interferometry, among others. Potential applications are x-ray imaging, neutron interrogation of substances, and neutrography.

\section{CONCLUSIONS}

Three kinds of pinch discharges driven by small devices were presented. The devices are relatively easy to construct and do not require large budgets, being suitable for small plasma laboratories.

The capillary discharge is a clever method to get high power densities with low energy. The experiment raises interesting technological questions that have not yet been answered: Is there an optimum combination of current derivative and peak current to obtain population inversion in the soft x-ray region, and if so, is it experimentally achievable?

Gas embedded Z-pinch experiments are useful to study pinch dynamics and instabilities. Peak currents of tens of kA, with current derivatives of $10^{12}-10^{13} \mathrm{~A} / \mathrm{s}$ could be achieved in small devices. Experiments in small Z-pinches are interesting to study the earliest stages of plasma discharges. However, diagnostics with sub-nanoseconds resolution within tens of micrometers are still required.

Neutron generation from plasma-focus devices has been a matter of research in the past. Nowadays, the technology is focused on transportable neutron generators for in situ applications (neutron illumination, neutrography, and substance detection). Moreover, intense neutron pulses have interesting applications in biology and material sciences. Plasma-focus pulses also show other unique technological features, namely simultaneous combination of $\mathrm{x}$-ray and neutron emissions, which can be used to study compound mixing of high and low atomic numbers. On the other hand, it is interesting to study if the existing theoretical models are valid in the very low energy limits. Although small plasma devices are relatively easy to construct, plasma diagnostics remain difficult. High spatial and temporal resolution diagnostics are required. In order to obtain the maximum benefit from these small devices, proper instruments and diagnostics must be developed.

Two of the described experiments were constructed in the Comisión Chilena de Energía Nuclear, CCHEN. In order to study the generated plasmas, the following diagnostics were developed and applied: fast voltage, current and current derivative monitor; time and space resolved pinhole images; time resolved VUV spectroscopy; and time integrated multipinhole x-ray photography. To the above diagnosis capability achieved at the CCHEN, we will add in the near future: 1 ns time resolution holographic interferometry with a $\mathrm{N}_{2}$ la- 
ser; two-dimensional optical multichannel analyzer; time integrated and temporal resolution neutrons detection; time resolved broad band emission using filtered PIN diode arrays; and time resolved $\mathrm{x}$-ray spectroscopy. Also, a small generator will be constructed, in order to study the initial stage of Z-pinch discharges.

The combination of the proposed diagnostics will allow us to perform accurate measurements of plasma dynamics, radiation, and particle emission. As an overall result, valuable experimental information will be available in order to be compared with theoretical models for dense plasmas.

\section{ACKNOWLEDGMENTS}

The authors acknowledge the fruitful discussions with $\mathrm{H}$. Chuaqui of Pontificia Universidad Católica de Chile; C. Moreno of Universidad de Buenos Aires (Argentina), $\mathrm{H}$. Bruzzone of Universidad de Mar del Plata (Argentina); K. N. Koshelev and P. Antsiferov of Institute of Spectroscopy, Russian Academic of Science, Troitsk; L. Altamirano of Dicontek, Chile, L. Huerta of Incite and Asociación para el Avance de las Ciencias (AAC, Chile), and D. Tótoro of Ceibo Produciones, Chile. The authors are very grateful for the insightful comments and suggestions of N. Pereira of Ecopulse (USA) and of an unknown referee.

This work has been funded by the grant Cátedra Presidencial en Ciencias, awarded to L. Soto by the Chilean government, Grant No. 1980187 Fondo Nacional de Investigación Científica y Tecológica (Chile), and Bilateral Agreement: Comisión Nacional de Energía Atómica (Argentina)_Comisión Chilena de Energía Nuclear (Chile).

${ }^{1}$ D. D. Ryutov, M. S. Derzon, and M. K. Matzen, "The physics of fast Z-pinches,' Rev. Mod. Phys. 72, 167 (2000).

${ }^{2}$ R. B. Spielman, C. Deeney, G. A. Chandler et al., Phys. Plasmas 5, 2105 (1998).

${ }^{3}$ P. S. Antsiferov, L. A. Dorokhin, E. Yu. Khautiev, Yu. V. Sidelnikov, D. A. Glushkov, I. V. Lugovenko, and K. N. Koshelev, J. Phys. D 31, 2013 (1998).

${ }^{4}$ J. J. Rocca, V. Shlyaptsev, F. G. Tomasel et al., Phys. Rev. Lett. 73, 2192 (1994).

${ }^{5}$ C. H. Moreno, M. C. Marconi, V. N. Shlyaptsev, B. R. Benware, C. D. Macchietto, J. L. A. Chilla, and J. J. Rocca, Phys. Rev. A 58, 1509 (1998).

${ }^{6}$ P. Choi, J. G. Lunney, A. Engel, C. Dumitrescu-Zoita, T. N. Hansen, I. Krisch, and J. Larour, J. Rous, "A $10^{13} \mathrm{~A} / \mathrm{s}$ high energy density microdischarge radiation source," Proceedings of the IEEE Pulse Power Confer- ence (Institute of Electrical and Electronics Engineers, Piscataway, NJ, 1999), p. 287.

${ }^{7}$ P. Choi and M. Favre, Rev. Sci. Instrum. 69, 3118 (1998).

${ }^{8}$ P. Choi, H. Chuaqui, M. Favre, and E. Wyndham, IEEE Trans. Plasma Sci. 15, 428 (1987).

${ }^{9}$ H. Chuaqui, M. Favre, L. Soto, and E. Wyndham, IEEE Trans. Plasma Sci. 21, 778 (1993).

${ }^{10}$ K. N. Koshelev (private communication, Troitsk, July 2000).

${ }^{11}$ A. Esaulov, P. Sasorov, L. Soto, M. Zambra, and J. Sakai, Plasma Phys. Controlled Fusion 43, 571 (2001).

${ }^{12}$ R. F. Post, Rev. Mod. Phys. 28, 338 (1956).

${ }^{13}$ P. Choi, M. Coppins, A. E. Dangor, and M. B. Favre, Nucl. Fusion 28, 1771 (1988)

${ }^{14}$ J. Sethian, A. E. Robson, K. A. Gerber, and A. W. De Silva, Phys. Rev. Lett. 59, 892 (1987)

${ }^{15}$ L. Soto, Ph.D. thesis, Pontificia Universidad Católica de Chile, Santiago de Chile, 1993.

${ }^{16}$ H. Chuaqui, L. Soto, M. Favre, and E. Wyndham, in Proceedings of the 3rd International Conference on Dense Z-Pinches, London, UK, 1993, AIP Conf. Proc., edited by M. Haines (American Institute of Physics, Woodbury, NY, 1993), p. 27.

${ }^{17}$ L. Soto, H. Chuaqui, M. Favre, and E. Wyndham, Phys. Rev. Lett. 72, 2891 (1994)

${ }^{18}$ L. Soto, H. Chuaqui, M. Favre, R. Saavedra, E. Wyndham, M. Skowronek, P. Romeas, R. Aliaga-Rossel, and I. Mitchell, IEEE Trans. Plasma Sci. 24, 1162 (1998).

${ }^{19}$ L. Soto, H. Chuaqui, and M. Skowronek, Appl. Opt. 34, 7831 (1995).

${ }^{20}$ A. E. Robson, Nucl. Fusion 28, 2171 (1988).

${ }^{21}$ M. G. Haines, Plasma Phys. Controlled Fusion 31, 759 (1989).

${ }^{22}$ M. G. Haines and M. Coppins, Phys. Rev. Lett. 66, 1462 (1991).

${ }^{23}$ T. D. Arber, M. Coppins, and J. Scheffel, Phys. Rev. Lett. 72, 2399 (1994).

${ }^{24}$ T. D. Arber, P. G. Russell, M. Coppins, and J. Scheeffel, Phys. Rev. Lett. 74, 2698 (1995).

${ }^{25}$ L. Soto, H. Chuaqui, M. Favre, and E. Wyndham, in Proceedings of the International Conference on Plasma Physics, ICPP, 1994, Foz do Iguazú, Brazil (American Institute of Physics, Woodbury, NY, 1994), p. 216.

${ }^{26}$ A. Esaulov, P. Sasorov, L. Soto, and M. Zambra, Phys. Plasmas 8, 1395 (2001).

${ }^{27}$ G. Decker, W. Kies, M. Mälzig, C. Van Valker, and G. Ziethen, Nucl. Instrum. Methods Phys. Res. A 249, 477 (1986).

${ }^{28}$ R. Aliaga-Rossel and P. Choi, IEEE Trans. Plasma Sci. 26, 1138 (1998); C. M. Ng, S. P. Moo, and C. S. Wong, ibid. 26, 1146 (1998).

${ }^{29}$ M. Favre, P. Silva, P. Choi, H. Chuaqui, C. Dumitrescu-Zoita, and E. S. Wyndham, IEEE Trans. Plasma Sci. 26, 1154 (1998).

${ }^{30}$ S. Lee, T. V. Tou, S. P. Moo, M. A. Eissa, A. V. Golap, K. H. Kewk, S. Mulyodrone, A. J. Smith, Suryad, W. Usada, and M. Zakaullah, Am. J. Phys. 56, 62 (1988).

${ }^{31}$ L. Soto and L. Altamirano, Rev. Sci. Instrum. 70, 1891 (1999).

${ }^{32} \mathrm{C}$. H. Moreno, H. Bruzzone, J. Martinez, and A. Clausse, IEEE Trans. Plasma Sci. 28, 1735 (2000). 